\title{
FOLFOX4 as First Line Treatment in Advanced Hepatocellular Cancer, when Sorafenib is not Available: Can it be an Option?
}

\author{
Piyush Vyas ${ }^{1 *}$, Marta Dudek¹, Ewa Zurawinska Grzelka ${ }^{1}$, Mateusz Cieslak ${ }^{1}$, Anna Dryja ${ }^{1}$ and Seema Vyas $^{2}$ \\ ${ }^{1}$ Medical University of Warsaw, Poland \\ ${ }^{2}$ Maria Sklodowska Curie Memorial Institute and Centre of Oncology, Poland
}

Submission: May 10, 2016; Published: May 30, 2016

*Corresponding author: Piyush Vyas, Consultant Clinical Oncologist; Dept. of Hematology, Oncology and Internal Medicine, Medical university of Warsaw Hospital, Banacha 1a Street, Warsaw, Poland, Email: vyas_piyush20@yahoo.co.in

\begin{abstract}
Protocol FOLFOX4 is a promising treatment option in advanced hepatocellular cancer patients as shown by Asian phase III trial - the EACH study. Given that most of the targeted therapies have not proven to be clinically significant or clinically relevant in the treatment advanced hepatocellular cancer, only sorafenib is at present an approved option the world over. Since sorafenib is not an affordable option, especially in developing countries where the burden is highest or for those who cannot be qualified for sorafenib in the western world, what are other options. After a thorough literature review it seems that if not Sorafenib, then probably we can treat patients with advanced hepatocellular cancer with chemotherapy protocol FOLFOX4. FOLFOX4 protocol has proved its safety and efficacy among advanced hepatocellular cancer patients in a phase III randomized trial - the EACH trial. EACH trial showed an improved PFS, RR, an increased tendency to improve OS among Asian patients: although categorically improved OS cannot be concluded.
\end{abstract}

Keywords: Hepatocellular Cancer; EACH; FOLFOX4; Sorafenib; Regorafenib; Sharp; Resourse

Abbreviations: HCC: Hepatocellular Cancer; ITT; Intent to Treat; RR: Response Rate; PFS: Progression Free Survival; OS: Overall Survival; DCR: Disease Control Rate; TACE: Trans Arterial Chemoembolisation; TAE: Trans Arterial Embolisation; NASH: Non Alcoholic Steatoheaptitis

\section{Introduction}

Hepatocellular cancer is third most common cause of cancer mortality and fifth most common cancer worldwide. Majority of the advanced hepatocellular cancer are in South East Asia , Western Africa where incidence is more than 15/100000 population, in Europe the incidence is $2 / 100000$ population. As far as etiology is concerned it is aflatoxin and Hepatitis $B$ virus in Asia and Africa. In US and in Southern Europe the majority of cases are caused by etiological agents like Hepatitis $\mathrm{C}$ virus, alcohol, non alcoholic fatty liver disease. Hepatocellular cancer is vaccine preventable disease, mass scale nationwide Hepatitis B vaccination programs for newborns conducted in Taiwan has shown promising results.HCV virus is transmitted by blood or plasma products, and risk of HCC due to HCV virus infection gets potentiated if co-factors like obesity, diabetes, alcohol intake and non alcoholic fatty liver co-exist. There seems to be rising trend of HCC due to alcoholic cirrhosis and
NASH (non alcoholic steatohepatis) in the western world. As far as symptoms are concerned include right upper quadrant pain, weight loss, early satiety. As far as diagnosis is concerned it is based on clinical, laboratory (AFP levels) and imaging. The most accepted staging systems are Cancer of Liver Italian Program (CLIP) and the Barcelona - Clinic Liver Cancer staging (BCLC) are based on performance status, tumor extent and liver function. However none of these staging systems have received universal acceptance, these staging systems provide best prognostic stratification of patients with HCC. Curative treatments: liver transplantation is the only definitive for HCC and underlying disease (cirrhosis). Surgical resection and ablative therapies especially radiofrequency ablative are some of the important therapies used for curative lesions. Palliative treatments include transarterial chemoembolization (TACE) and transarterial embolisation (TAE). Among systemic therapies only sorafenib a targeted therapy has shown an increased OS of 


\section{Cancer Therapy \& Oncology International Journal}

3 months as shown by SHARP trial among Child Pugh A patients with advanced HCC. Recently Regorafenib, a targeted therapy has shown promising results in a phase III randomized clinical trial as a second line therapy among advanced unresectable HCC patients after treatment with sorafenib as first line treatment [1-4].

\section{Systemic Therapies In Advanced Hepatocellular Cancer}

Advanced HCC cancer has seen a lot of failures. Trials with many targeted therapies except with Sorafenib have proved to be negative trials. For example codrituzumab [5], Linifarib, Sunitinib, Brivanib , cixutumumab [6-9], Everolismus showed no significant increase in overall survival (OS). Only Sorafenib as shown in the SHARP (1) trial in the West and as shown in Oriental trial conducted in the East showed statistically significant (3 months) increase in OS [10-14].

Recently RESOURSE trial - a phase III trial has shown that regorafenib has met it's primary endpoint of a statistically improved overall survival in patients with unresectable HCC whose disease has progressed after treatment with Sorafenib [15]. Thus there exists a hope of getting a second line therapy registered for advanced unresectable HCC.

Since most of the targeted therapies investigated till now have not proved to be better than the existing standard -Sorafenib, a genuine interest has been drawn towards finding effective chemotherapy regimen which either alone or in combination with targeted therapies can be used as a treatment option. FOLFOX4 has proved to be a promising regimen as shown in Asian EACH study [2]. In EACH trial the study did not meet it's primary endpoint but showed a trend towards improved OS with FOLFOX4 and also showed an increased RR and PFS among Asian patients. Although a clear OS benefit could not be concluded from EACH trial but this trial seems to be an important trial.

\section{Rationale Behind Treatment of Advanced HCC with FOLFOX4 Protocol}

HCC has high incidence in China, Western Africa, Eastern Africa, and South East Asia, significant number of cases occur in Carribean, Micropolynesia and in South Africa. In many of these countries the median survival is poor, for example less than six months in Western Africa. Since most of the cases occur in economically backward countries, there exists a need for an affordable treatment option.In West and the world over, although Sorafenib has been approved to be the only treatment option, many patients do not get their therapies financed due to restrictive inclusion criteria of the funding agencies. HCC incidence has been increasing in the west due to chronic Hepatitis C infection, excessive alcohol consumption, obesity and NASH. Thus with the incidence and number of HCC patients increasing, the burden of the disease will increase on the funding agencies. Funding agencies thus would be able to fund Sorafenib or Regorafenib for every advanced HCC cancer patient even in economically developed countries.

Many patients have significant hepatic dysfunction, hepatic impairment thus can be rendered unsuitable for treatment with regorafenib. Patients with malignant hypertension and other serious cardiological conditions are generally disqualified for treatment with Sorafenib. Thus it seems that there exists a need of much affordable alternate option so that majority of HCC patients can get at least some type of safe and effective treatment, if not the ideal one.

\section{EACH Study}

EACH study included patients with advanced HCC who were ineligible for surgery or localized treatments where systemic chemotherapy was the only option. The rationale behind this study was that the most commonly administered chemotherapy protocol DCF- Doxorubicin, Cisplatin, 5 Florouracil offered no survival benefit. Since oxaliplatin containing regimens like GEMOX, XELOX and FOLFOX4 have shown clinical activity in advanced HCC phase II studies, oxaliplatin containing regimen appeared to be genuine protocol for further studies. The reason behind using doxorubicin as a comparator was that it was most effective regimen at the time of study (2006-2007).

EACH trial (2) was a phase III open label randomized study conducted in mainland China, Taiwan, Korea and Thailand which included 371 patients. 184 patients were on FOLFOX4 arm and 187 patients were on Doxorubicin arm. The treatment was continued until progression, toxicity, death or until patient was rendered eligible for resection. As far as objectives of this study is concerned, the primary endpoints were to determine whether FOLFOX4 improves overall survival (OS) compared to doxorubicin. The secondary endpoint was to compare the efficacy and safety of the two treatment regimens to evaluate PFS, RR, DCR by RECIST. Also the safety criteria were seen as per NCI-CTC AE v 3.0.

As far as inclusion criteria are concerned, the inclusion criteria were advanced and/or unrespectable HCC; at least one measurable lesion (on CT, more than or equal to $2 \mathrm{~cm}$, on spiral CT or MRI more than or equal to $1 \mathrm{~cm}$ ). The study included patients who were cancer treatment naive (except for surgery) or progressed after previous intervention TACE/ TAE / local therapy. Other inclusion criteria were adequate organ and bone marrow function i.e., Child Pugh stage A/B. As per BCLC stage B/C and Karnofsky at least $70 \%$. The exclusion criteria were: previous liver transplantation, CNS metastasis, previous oxaliplatin /doxorubicin treatment, use of other anti cancer therapies other than radiotherapy to non target lesion. Also drugs including IFN alpha or other traditional herbal medicines were exclusion criteria.

EACH study (2) showed that FOLFOX4 regimen has better OS in ITT (intended to treat) population. It showed median OS of 6, 4 
months in FOLFOX4 arm vs 4.97 months in doxorubicin arm. The OS benefit with FOLFOX4 was very similar to OS with sorafenib in Asian population (Oriental study). Sorafenib showed OS of 6.5 months, FOLFOX4 showed 6.4 months.

The overall survival in Chinese population was much better - m OS benefit of 5,7 months with FOLFOX4 vs 4, 3 months with doxorubicin which was significant on continued follow up $(\mathrm{p}=0,028)$. The overall survival was not good if a patient had a poorly differentiated HCC, and those who had grade $3 / 4$ as per NCI/CTC criteria toxicities. The secondary endpoints in ITT population were median PFS-2, 93 months vs 1, 77 months. The FOLFOX4 regimen significantly increased DCR and RR vs Doxorubicin: RR-8,2\% VS 2,7\% .DCR $-52,2 \%$ vs $31,6 \%$.

So there exists a need to conduct prospective clinical trials with FOLFOX4 regimens in advanced hepatocellular cancer patients. Since pharmacogenetic variations do occur, it seems that there exists a need to validate the data provided by the east.

\section{FOLFOX4 protocol}

In the light of new data provided by EACH trial - a multicentre, multinational, randomized phase 3 trial oxaliplatin containing protocols have generated a lot of interest, especially FOLFOX4 protocol. It seems that for patients with advanced hepatocellular cancer who have no contraindications for systemic chemotherapy FOLFOX4 regimen can prove to be better option than best supportive care. The future probable indications for FOLFOX4 protocol in advanced hepatocellular carcinoma can be patients with extrahepatic metastasis, patients disqualified for TACE/TAE, patients having tumor thrombi in vena cava inferior; patients having thrombi in portal vein trunk, those having liver vascular obstruction following after several TACE /TAE therapy and patients where recurrence is observed after interventional treatment. Chinese guidelines have approved oxaliplatin containing protocols (FOLFOX4) for systemic therapy in advanced HCC. Chinese guideline have clearly stated that systemic therapy with oxaliplatin containing protocols are superior to supportive/ symptomatic treatment given the patient do not have contraindications to chemotherapy(ECOG $>2$; Child Pugh $>7$;WBC $<3$ thousand per uL.; Neutrophiles $<1,5$ thousand per uL.;PLT- $<60$ thousand/uL.; Hb. $<9,0$ g/dl.; AST/ALT $>5$ times upper limit; bilirubin $>2$ times the upper limit; serum albumin $<28 \mathrm{~g} / \mathrm{L}$; creatinine if more than upper limit; creatinine clearance rate less than $50 \mathrm{ml} / \mathrm{min}$., hepatic encephalopathy, bleeding tendencies, patients having infections, also if patient have intermediate to large amount of peritoneal effusion).

\section{Discussion}

Since many targeted therapies except Sorafenib and regorafenib, evaluated in various clinical trials have given negative results, a genuine interest has been created in classical chemotherapy regimens like FOLFOX4. Researchers and clinicians have been even trying to combine both sorafenib and classical chemotherapy treatment options. Sorafenib in combination with chemotherapy (doxorubicin) as shown by Abou-Alfa et al. [5] has shown promising results. Abou Alfa et al. [7] conducted a study examining the efficacy and safety of sorafenib with doxorubicin in patients with advanced HCC, and followed that median survival was 13,7 months (95\%CI 8,9-not reached) was attained in comparison to 6,5 months $(4,5-9,9)$ among patients treated with doxorubicin alone $(p=0,006)$.

Thus it seems there exists an urgent need to do prospective trials in the west with FOLFOX4 protocol alone or in combination with Sorafenib as first line treatment in advanced hepatocellular cancer patients who do not qualify for loco-regional therapies or are not suitable for resection. Advanced hepatocellular cancer population in the west has different etiology (Hepatitis C virus, alcohol, non alcoholic steatohepatis etc.), thus data provided by EACH study needs validation in the west. It seems the future lies in searching effective chemotherapy protocols and targeted therapies that could synergize or potentiate the antiproliferative activity.

\section{Conclusion}

The only approved drug in the treatment of advanced hepatocellular cancer is a multikinase inhibitor Sorafenib which improves overall survival by only 3 months as shown by SHARP trial. And chemotherapy protocol which has shown better RR and PFS, but not OS (however a tendency for increased OS observed) for advanced HCC patients among Asian patients is FOLFOX4 as shown by Qin S et al. [2,4]. The need of the hour is to investigate FOLFOX4 protocol alone and in combination with Sorafenib in the western patients with advanced HCC. As the HCC disease burden is mostly on the developing world where availability of Sorafenib may be difficult, FOLFOX4 protocol thus remains an affordable economic option if accepted as an effective treatment worldwide. China Food and drug administration has accepted FOLFOX4 as an effective treatment option among advanced HCC patients, taking a lead. Probably at present FOLFOX4 protocol remains the best affordable option for patients not rendered suitable for sorafenib or regorafenib the world over.

\section{References}

1. Llovet JM, Ricci S, Mazzaferro V, Hilgard P, Gane E, et al. (2008) Sorafenib in advanced hepatocellular carcinoma. N Engl J Med 359(4): 378-390.

2. Qin S, Bai Y, Lim HY, Thongprasert S, Chao Y, et al. (2013) Randomised, multicellular, open label study of oxaliplatin plus fluorouracil / leucovorin vs doxorubicin as palliative chemotherapy in patients with advanced hepatocellular cancinoma from Asia. J Clin Oncol 31(28): 3501-3508.

3. Phase 2 trial of Sorafenib in combination with modified FOLFOX4 in patients with advanced hepatocellular cancer. Study type interventional. Andrew X Zhu. Massachusetts General Hospital, USA.

4. Qin S. (2013) Guidelines on the diagnosis and treatment of primary liver cancer (2011 edition). Chin Clin Oncol 1(1): 01-10.

5. Abou-Alfa GK, Puig, Daniele B, Kudo M, Merle P, et al. (2016) Randomized Phase II placebo controlled study of codrituzumab in previously treated patients with advanced hepatocellular carcinoma. J Hepatol S0168-8278(16): 30106-30114. 


\section{Cancer Therapy \& Oncology International Journal}

6. Abou-Alfa GK. (2009) Selection of patients with hepatocellular carcinoma for sorafenib. J Natl Compr Canc Netw 7(4): 397-403.

7. Abou-Alfa GK, Johnson P, Knox JJ, Capanu M, Davidenko I, et al. (2010) Doxorubicin plus sorafenib vs doxorubicin alone in patients with advanced hepatocellular carcinoma: a randomized trial. JAMA 304(19): 2154-2160.

8. Abou-Alfa GK (2013) Hepatocellular carcinoma: what is happening everywhere? A phase II study of cixutumumab (IMC-A12, NSC742460) in advanced hepatocellular carcinoma. Chin Clin Oncol 2(4):31.

9. Abou-Alfa GK, Capanu M, O’Reilly EM, Ma J, Chou JF et al. (2014) A phase II study of cixutumumab (IMC-A12, NSC742460) in advanced hepatocellular carcinoma. J Hepatol 60(2):319-324.

10. Kelley RK (2013) Brivanib and FOLFOX in Hepatocellular Carcinoma: Finding the Common Themes Among Negative Trials. J Clin Oncol 31(28): 3483-3486
11. Ying-Chun Shen, Zhong-Zhe Lin, Chih-Hung Hsu, Chiun Hsu, Yu-Yun Shao, et al. (2013) Clinical Trials in Hepatocellular Carcinoma: An Update. Liver Cancer 2(3-4): 345-364.

12. Chan SL (2014) Drug Development for Hepatocellular Carcinoma: Knowing the Past Helps to Understand the Future. Oncologist 19(11): 1115-1117.

13. Hua Zhu, Ping Sun (2012) Clinical study of FOLFOX4 regimen for patients of advanced hepatocellular carcinoma. Chinese German Journal of Clinical Oncology 11(3): 34-137.

14. Bayer: Science For A Better Life. USA.

15. Bayer (2016) Phase III trial of Regorafenib in patients with unresectable liver cancer meets primary endpoint of improving survival. 13: 26 . 